\title{
A KERNEL APPROACH TO THE LOCAL SOLVABILITY OF THE TANGENTIAL CAUCHY RIEMANN EQUATIONS
}

BY

\author{
A. BOGGESS ${ }^{1}$ AND M.- C. SHAW
}

\begin{abstract}
An integral kernel approach is given for the proof of the theorem of Andreotti and Hill which states that the $Y(q)$ condition of Kohn is a sufficient condition for local solvability of the tangential Cauchy Riemann equations on a real hypersurface in $\mathbf{C}^{n}$. In addition, we provide an integral kernel approach to nonsolvability for a certain class of real hypersurfaces in the case when $Y(q)$ is not satisfied.
\end{abstract}

1. Let $M$ be a smooth real hypersurface in $\mathbf{C}^{n}$ and let $z_{0} \in M$. If $q$ is an integer with $0 \leq q \leq n-1$, then we say that $M$ satisfies condition $Y(q)$ (cf. [FK]) at $z_{0}$ if the Levi form of $M$ at $z_{0}$ has either $\max \{n-q, q+1\}$ eigenvalues of the same sign or $\min \{n-q, q+1\}$ positive and $\min \{n-q, q+1\}$ negative eigenvalues. Note that if the Levi form of $M$ at $z_{0}$ has $r$ positive and $s$ negative eigenvalues and $r+s=n-1$, then $M$ satisfies condition $Y(q)$ for all $0 \leq q \leq n-1$ except $q=r, s$. In particular, if $M$ is strictly pseudoconvex, $M$ satisfies condition $Y(q)$ for all $1 \leq q \leq n-2$. In 1972, Andreotti and Hill [AH], showed that if $M$ satisfies condition $Y(q)$ at $z_{0}$, then the tangential Cauchy Riemann equations $\left(\bar{\partial}_{M} u=f\right)$ are locally solvable in degree $q$ near $z_{0}$. It has been the goal of recent research to represent a solution $u$ by integral formulas. This was done by Henkin [He, Theorem 5.1] in 1977 in the strictly pseudoconvex case. Integral formulas for $u$ were also found in 1980 in [B2] under the assumption that $M$ satisfies the first half of condition $Y(q)$. In this paper, we handle the latter half of condition $Y(q)$ and thus we provide an entirely integral kernel approach to the proof of the result of Andreotti and Hill mentioned above. In addition, we provide an integral kernel approach to nonsolvability when condition $Y(q)$ is not satisfied. Finally, if $q=0$, our approach yields (yet) another proof of the classical Hans Lewy (2 sided) CR extension theorem with an integral kernel representation of the extension.

To state our result let us introduce some notation. $\Lambda^{p, q}\left(\mathbf{C}^{n}\right)$ will denote the bundle of $(p, q)$ forms on $\mathbf{C}^{n}$. If $V$ is an open set in $\mathbf{C}^{n}$, then $\mathscr{E}^{p, q}(V)$ (resp. $\left.\mathscr{D}^{p, q}(V)\right)$ will denote the space of smooth sections of $\Lambda^{p . q}\left(\mathbf{C}^{n}\right)$ over $V$ (resp. with compact support in $V$ ). Let $M$ be a smooth real hypersurface defined by $M=\left\{z \in \mathbf{C}^{n} ; \rho(z)=0\right\}$, where $\rho: \mathbf{C}^{n} \rightarrow \mathbf{R}$ with $d \rho \neq 0$ on $M$. Let $\Lambda^{p, q}(M)$ be the subbundle of $\left.\Lambda^{p, q}\left(\mathbf{C}^{n}\right)\right|_{M}$ consisting of those forms in $\left.\Lambda^{p, q}\left(\mathbf{C}^{n}\right)\right|_{M}$ which are orthogonal to the ideal generated by $\bar{\partial} \rho(z), z \in M$ (under the usual Hermitian metric for forms in $\mathbf{C}^{n}$ ). If $V$ is an open

\footnotetext{
- Received by the editors June 16, 1984.

1980 Mathematics Subject Classification. Primary 35C15, 35N15, 32D10, 32E99, 32F99.

${ }^{1}$ This author was partially supported by NSF grant \# MCS-8301369.
} 
subset of $M$, we let $\mathscr{E}_{M}^{p} \cdot q(V)\left(\right.$ resp. $\left.\mathscr{D}_{M}^{p \cdot q}(V)\right)$ denote the space of smooth sections of $\Lambda^{p, q}(M)$ over $V$ (resp. with compact support in $V$ ).

We let $T_{M}:\left.\Lambda^{p . q}\left(\mathbf{C}^{n}\right)\right|_{M} \rightarrow \Lambda^{p . q}(M)$ be the orthogonal projection map. With this notation, $\bar{\partial}_{M}: \mathscr{E}_{M}^{p, q}(V) \rightarrow \mathscr{E}_{M}^{p, q+1}(V)$ is defined as follows. Suppose $f \in \mathscr{E}_{M}^{p, q}(V)$. Let $\tilde{f} \in \mathscr{E}^{p . q}\left(\mathbf{C}^{n}\right)$ with $\left\{\left.\tilde{f}\right|_{V}\right\}_{T_{M}}=f$. Then $\bar{\partial}_{M} f:=\left\{\left.\bar{\partial} \tilde{f}\right|_{V}\right\}_{T_{M}}$.

Our goal in this paper is to provide integral kernel proofs of the following theorems.

1.1. TheOREM. Suppose $M$ is a smooth real submanifold of $\mathbf{C}^{n}$ and $z_{0}$ is a point in $\mathbf{M}$. Suppose $M$ satisfies condition $Y(q)$ at $z_{0}$ for some integer $q, 1 \leq q \leq n-1$. Given an open set $\omega \ni z_{0}$ in $M$, there is an open set $\omega^{\prime} \ni z_{0}$ in $M$ with $\omega^{\prime} \subset \omega$ such that if $f \in \mathscr{E}_{M}^{p, q}(\omega)$ with $\bar{\partial}_{M} f=0$ on $\omega$, then there is a $u \in \mathscr{E}_{M}^{p, q-1}\left(\omega^{\prime}\right)$ with $\bar{\partial}_{M} u=$ fon $\omega^{\prime}$.

DEFINITION. We say that the tangential Cauchy Riemann equations are not locally solvable in degree $q$ in $z_{0}$ if there is a fundamental sequence of open sets $\{\omega\}$ such that for each $\omega$ there is an $f \in \mathscr{E}^{p, q}(\omega)$ with $\bar{\partial}_{M} f=0$ on $\omega$ for which there is no smooth $u$ which solves $\bar{\partial}_{M} u=f$ in any neighborhood of $z_{0}$.

1.2 Theorem (Nonsolvability [Hi]). Suppose the Levi form of $M$ at $z_{0}$ has $q$ positive and $n-q-1$ negative eigenvalues for some integer $q, 1 \leq q \leq n-1$. Then the tangential Cauchy Riemann equations are not locally solvable in degrees $q$ and $n-q-1$ at $z_{0}$.

Note that conditions $Y(q)$ and $Y(n-q-1)$ are not satisfied in the above theorern. Actually the hypothesis can be weakened somewhat to include examples of hypersurfaces with degenerate Levi forms which Hill was not able to handle in [Hi] (cf. §5).

1.3 Theorem (HANS LeWy's 2 - SIDED EXTENSION THEOREM [L]). Suppose the Levi form of $M$ at $z_{0}$ has at least one positive and one negative eigenvalue (condition $Y(0)$ ). Given $\omega \ni z_{0}$ an open set in $M$, there exists an open set $W \ni z_{0}$ in $\mathbf{C}^{n}$ such that if $f \in \mathscr{E}_{M}^{p .0}(\omega)$ with $\bar{\partial}_{M} f=0$ on $\omega \cdot(C R$ function $)$, then there is a unique $F \in \mathscr{E}^{p .0}(W)$ with $\bar{\partial} F=0$ on $W$ and $\left.F\right|_{\omega}=f$.

We also note there are related recent results of Treves [T1, T2] which discuss solvability and nonsolvability of overdetermined systems of partial differential equations with real analytic coefficients. His techniques do not involve kernels and are quite different from ours.

2. Let $u, v: \mathbf{C}^{n} \times \mathbf{C}^{n} \rightarrow \mathbf{C}^{n}, u(\zeta, z)=\left(u_{1}(\zeta, z), \ldots, u_{n}(\zeta, z)\right), v(\zeta, z)=$ $\left(v_{1}(\zeta, z), \ldots, v_{n}(\zeta, z)\right)$, be smooth functions. We shall use the notation

$$
\begin{aligned}
u(\zeta, z) \cdot(\zeta-z) & =\sum_{j=1}^{n} u_{j}(\zeta, z)\left(\zeta_{j}-z_{j}\right), \\
u(\zeta, z) \cdot d(\zeta-z) & =\sum_{j=1}^{n} u_{j}(\zeta, z) d\left(\zeta_{j}-z_{j}\right), \\
\bar{\partial} u(\zeta, z) \cdot d(\zeta, z) & =\sum_{j=1}^{n} \bar{\partial} u_{j}(\zeta, z) \wedge d\left(\zeta_{j}-z_{j}\right),
\end{aligned}
$$


and we define the following kernels:

$$
L(u)(\zeta, z)=(2 \pi i)^{-n}\left[\frac{u(\zeta, z) \cdot d(\zeta-z)}{u(\zeta, z) \cdot(\zeta-z)}\right] \wedge\left[\frac{\bar{\partial} u(\zeta, z) \cdot d(\zeta-z)}{u(\zeta, z) \cdot(\zeta-z)}\right]^{n-1}
$$

$$
L(v)(\zeta, z) \quad(\text { defined as above with } u \text { replaced by } v) \text {, }
$$

$$
\begin{aligned}
R(u, v)(\zeta, z)= & (2 \pi i)^{-n}\left[\frac{u(\zeta, z) \cdot d(\zeta-z)}{u(\zeta, z) \cdot(\zeta-z)}\right] \wedge\left[\frac{v(\zeta, z) \cdot d(\zeta-z)}{u(\zeta, z) \cdot(\zeta-z)}\right] \\
& \wedge \sum_{j+k=n-2}\left[\frac{\bar{\partial} u(\zeta, z) \cdot d(\zeta-z)}{u(\zeta, z) \cdot(\zeta-z)}\right]^{j} \wedge\left[\frac{\bar{\partial} v(\zeta, z) \cdot d(\zeta-z)}{v(\zeta, z) \cdot(\zeta-z)}\right]^{k}
\end{aligned}
$$

The $\bar{\partial}$ operator used in the above formulas is taken with respect to both variables $\zeta$ and $z$. If we wish to emphasize $\bar{\partial}$ with respect to say the variable $\zeta$, we shall write $\bar{\partial}_{\zeta}$. We have $\bar{\partial}_{\zeta, z}=\bar{\partial}_{\zeta}+\bar{\partial}_{z}$.

We have the identities (cf. [HP], Theorem 4.10])

$$
\begin{gathered}
\bar{\partial}_{\zeta, z}\{R(u, v)(\zeta, z)\}=L(v)(\zeta, z)-L(u)(\zeta, z), \\
\bar{\partial}_{\zeta, z}\{L(u)(\zeta, z)\}=\bar{\partial}_{\zeta, z}\{L(v)(\zeta, z)\}=0 .
\end{gathered}
$$

These identities hold on the sets where the kernels are smoothly defined.

Let $M$ be a smooth real and orientable hypersurface in $\mathbf{C}^{n}$. We shall eventually integrate the above kernels over $M$. To do so, we shall require the functions $u$ and $v$ to satisfy certain properties which we now discuss. Suppose $M$ is the boundary of an open set $D \subset \mathbf{C}^{n}$.

2.3. Definition. We say that a smooth function $\rho: \mathbf{C}^{n} \rightarrow \mathbf{R}$ is a defining function for the pair $(M, D)$ if (i) $\rho(\zeta)=0$ on $M$, (ii) $\rho(\zeta)<0, \zeta \in D$, and (iii) $d \rho(\zeta) \neq 0$ on $M$.

2.4. Definition. We say that $u$ is a local support function for $(M, D)$ at a point $z_{0} \in M$ if there exists an open set $U \ni z_{0}$ and a defining function $\rho$ for $(M \cap U$, $D \cap U$ ) such that:

(a) there exists a constant $C>0$ with

(2.4a) $\operatorname{Re}[u(\zeta, z) \cdot(\zeta-z)] \geq C\left[\rho(\zeta)-\rho(z)+|\zeta-z|^{2}\right] \quad$ for $\zeta, z \in U$

(b) for each $z \in U$

$$
\left.d_{\zeta}\{\operatorname{Im}[u(\zeta, z) \cdot(\zeta-z)]\}\right|_{\zeta=z} \wedge d \rho(z) \neq 0 .
$$

Now let $M$ divide $C^{n}$ into two open sets $D^{-}$and $D^{+}$and let $z_{0} \in M$. Suppose $u$ is a local support function for $\left(M, D^{-}\right)$at $z_{0}$ and let $v$ be a local support function for $\left(M, D^{+}\right)$at $z_{0}$. Note that if $\zeta \in M \cap U$ and $z \in D^{-} \cap U$ ( $U$ is the open set in Definition 2.4), then $u(\zeta, z) \cdot(\zeta-z) \neq 0$ by (2.4a). Therefore, if $f \in \mathscr{D}^{p, q}(M \cap U)$, then the form

$$
L(u)\left([M]^{0,1} \wedge f\right)(z):=\int_{\zeta \in M} \frac{n, n-1}{L(u)(\zeta, z) \wedge f(\zeta)}
$$


is well defined and smooth for $z \in U \cap D^{-}$. Here, the bracket above the integrand indicates that only the piece of type $(n, n-1)$ in $\zeta$ in the integrand is integrated over $M$. Likewise, the form $L(v)\left([M]^{0.1} \wedge f\right)(z)$ is well defined and smooth for $z \in U \cap D^{+}$. The next proposition allows us to take boundary values of $L(u)\left([M]^{0,1} \wedge f\right)(z)$ and $L(v)\left([M]^{0,1} \wedge f\right)(z)$ as $z$ approaches $M$ from $D^{-}$and $D^{+}$ respectively. It also allows us to define $R(u, v)\left([M]^{0,1} \wedge f\right)(z)$ for $z \in M$.

2.5 Proposition. Let $M$ divide $\mathbf{C}^{n}$ into the open sets $D^{+}$and $D^{-}$and let $z_{0} \in M$. Suppose $u$ is a local support function for $\left(M, D^{-}\right)$at $z_{0}$ and $v$ is a local support function for $\left(M, D^{+}\right)$at $z_{0}$. Let $f \in \mathscr{D}^{p, q}(M \cap U), 0 \leq q \leq n-1,0 \leq p \leq n$.

(a) $L(u)\left([M]^{0,1} \wedge f\right)$ is smooth $\left(C^{\infty}\right)$ on $\overline{D^{-}} \cap U$. In particular $L_{M}^{-}(u)(f):=\left\{\left.L(u)\left([M]^{0,1} \wedge f\right)\right|_{M}\right\}_{T_{M}}$ defines a smooth form on $M \cap U$.

(b) $L(v)\left([M]^{0,1} \wedge f\right)$ is smooth $\left(C^{\infty}\right)$ on $\overline{D^{+}} \cap U$. In particular $L_{M}^{+}(v)(f):=\left\{\left.L(v)\left([M]^{0.1} \wedge f\right)\right|_{M}\right\}_{T_{M}}$ defines a smooth form on $M \cap U$.

(c) $R(u, v)(\zeta, z)$ is locally integrable on $\{M \cap U) \times\{M \cap U\}$. Moreover

$$
R_{M}(u, v)(f)(z):=\left\{\int_{\zeta \in M} \stackrel{n, n-1}{R(u, v)(\zeta, z) \wedge f(\zeta)}\right\}_{T_{M}}
$$

defines a smooth form for $z \in M \cap U$.

(d) For $f \in \mathscr{D}^{p . q}(M \cap U), 0 \leq q \leq n-1$,

$$
\begin{aligned}
f= & -\left[\bar{\partial}_{M}\left\{R_{M}(u, v)(f)\right\}+R_{M}(u, v)\left(\bar{\partial}_{M} f\right)\right] \\
& +\left[L_{M}^{+}(v)(f)-L_{M}^{-}(u)(f)\right] \text { on } M \cap U .
\end{aligned}
$$

Parts (a) and (b) are proved in [HP2, Theorem 8.1]. Instead of (2.4b), they assume

$$
\left.\left.d_{\zeta}\{\operatorname{Re} u(\zeta, z) \cdot(\zeta-z)\}\right|_{\zeta=z} \wedge d_{\zeta}\{\operatorname{Im} u(\zeta, z) \cdot(\zeta-z)\}\right|_{\zeta=z} \neq 0
$$

for $z \in U$ and likewise for $v$. However the proof of (a) and (b) can be simplified by using $(2.4 \mathrm{~b})$ because $(2.4 \mathrm{~b})$ allows a smooth change of variables which flattens $M$ and declares $\operatorname{Im}(u(\zeta, z) \cdot(\zeta-z))$ to be an independent coordinate. In any event all support functions constructed here will satisfy both (2.4b) and (2.6). The regularity of the $R$ kernel is proved in [He, Theorem 3.1] (see also [B1, Theorem 3.8]). In these two references, $M$ is assumed to be strictly pseudoconvex. However, only the properties (2.4a) and (2.4b) are used in the proofs. Part (d) is proved in [He, Theorem 3.2] (see also [B2, §§3, 4] for a discussion which employs our notation).

Note from $(2.5 \mathrm{~d})$ that the terms $L_{M}^{+}(v)(f)$ and $L_{M}^{-}(u)(f)$ are obstructions to solving the equations $\bar{\partial}_{M} g=f$. If $M=\{z ; \rho(z)=0\}$, where $\rho: \mathbf{C}^{n} \rightarrow \mathbf{R}$ is a strictly convex function, and if $D^{+}=\{z ; \rho(z)>0\}$ and $D^{-}=\{z ; \rho(z)<0\}$, then it is a special case of Lemma 3.6 (with $t=n-1$ ) in the next section that the functions

$$
u_{j}(\zeta, z)=\frac{\partial \rho}{\partial \zeta_{j}}(\zeta), \quad v_{j}(\zeta, z)=\frac{-\partial \rho}{\partial z_{j}}(z), \quad 1 \leq j \leq n
$$

are local support functions at $z_{0}$ for $\left(M, D^{-}\right)$and $\left(M, D^{+}\right)$respectively. Note that in this case $u$ only depends on $\zeta$ and $v$ only depends on $z$. From (2.1a) and (2.1b) we see that the degree of $L(u)$ and $L(v)$ in $d \bar{\zeta}$ is $n-1$ and 0 respectively. Therefore 
$L_{M}^{-}(u)(f)=0$ unless $f \in \mathscr{D}^{p .0}(M \cap U)$ and $L_{M}^{+}(v)(f)=0$ unless $f \in$ $\mathscr{D}^{p, n-1}(M \cap U)$. Thus these terms are not obstructions when $f \in \mathscr{D}^{p, q}(M \cap U)$, $1 \leq q \leq n-2$. It will be the goal of the next section to construct local support functions for $M$ at $z_{0}$ assuming the condition $Y(q)$ holds at $z_{0}$. We shall construct $u$ and $v$ so that the terms $L_{M}^{-}(u)(f)$ and $L_{M}^{+}(v)(f)$ vanish when $f \in \mathscr{D}^{p, q}(M \cap U)$.

3. Before constructing the support functions, we need to construct defining functions for $M$ which have a particularly nice form. Let us assume that the given point $z_{0} \in M$ is the origin and that $M$ is graphed over its real tangent space at 0 , i.e.

$$
M=\left\{z \in \mathbf{C}^{n} ; \operatorname{Re} z_{n}=f\left(z_{1}, \ldots, z_{n-1}, \operatorname{Im} z_{n}\right)\right\} \quad \text { with } f(0)=0, \nabla f(0)=0 .
$$

Thus $f(z)=\mathcal{O}\left(|z|^{2}\right)$ which means that there is a uniform constant $C$ such that $|f(z)| \leq C|z|^{2}$ for all $z$ sufficiently small.

Let $\rho_{1}(z)=\operatorname{Re} z_{n}-f(z)$, so that $\rho_{1}=0$ on $M$. By replacing $\rho_{1}$ by $\rho_{1}+c\left(\rho_{1}\right)^{2}$ for an appropriate constant $c$, we can arrange $\left(\partial^{2} \rho_{1} / \partial z_{n} \partial \bar{z}_{n}\right)(0)=1$. A second order Taylor expansion of $\rho_{1}$ about 0 yields

$$
\begin{aligned}
\rho_{1}(z)= & \operatorname{Re} z_{n}+\operatorname{Re}\left[\sum_{j, k=1}^{n} \frac{\partial^{2} \rho_{1}}{\partial z_{j} \partial z_{k}}(0) z_{j} z_{k}\right]+\sum_{j, k=1}^{n-1} \frac{\partial^{2} \rho_{1}}{\partial z_{j} \partial \bar{z}_{k}}(0) z_{j} \bar{z}_{k}+\left|z_{n}\right|^{2} \\
& +2 \operatorname{Re}\left[\sum_{j=1}^{n-1} \frac{\partial^{2} \rho_{1}}{\partial z_{j} \partial \bar{z}_{n}}(0) z_{j} \bar{z}_{n}\right]+\mathcal{O}\left(|z|^{3}\right) .
\end{aligned}
$$

Note that

$$
\begin{aligned}
\operatorname{Re}\left[\sum_{j=1}^{n-1} \frac{\partial^{2} \rho_{1}}{\partial z_{j} \partial \bar{z}_{n}}(0) z_{j} \bar{z}_{n}\right]= & -\operatorname{Re}\left[\sum_{j=1}^{n-1} \frac{\partial^{2} \rho_{1}}{\partial z_{j} \partial \bar{z}_{n}}(0) z_{j} z_{n}\right] \\
& +2 \operatorname{Re}\left[\sum_{j=1}^{n-1} \frac{\partial^{2} \rho_{1}}{\partial z_{j} \partial \bar{z}_{n}}(0) z_{j}\right] \operatorname{Re} z_{n} .
\end{aligned}
$$

Now let $\rho$ be defined by the same formula for $\rho_{1}$ as above except that we replace $\operatorname{Re} z_{n}$ by $f(z)$ in the last term on the right of (3.1). Since $\operatorname{Re} z_{n}=f(z)$ for $z \in M$, clearly $\rho$ is also a defining function for $M$. Since $f(z)=\mathcal{O}\left(|z|^{2}\right)$, we have $\rho_{1}=\rho+$ $\mathcal{O}\left(|z|^{3}\right)$ and therefore

$$
\begin{aligned}
\rho(z)= & \operatorname{Re} z_{n}+\operatorname{Re}\left[\sum_{j, k=1}^{n} \frac{\partial^{2} \rho}{\partial z_{j} \partial z_{k}}(0) z_{j} z_{k}-2 \sum_{j=1}^{n-1} \frac{\partial^{2} \rho}{\partial z_{j} \partial \bar{z}_{n}}(0) z_{j} z_{n}\right] \\
& +\sum_{j, k=1}^{n-1} \frac{\partial^{2} \rho}{\partial z_{j} \partial \bar{z}_{k}}(0) z_{j} \bar{z}_{k}+\left|z_{n}\right|^{2}+\mathcal{O}\left(|z|^{3}\right) .
\end{aligned}
$$

We now make the analytic change of variables

$$
\begin{aligned}
& \hat{z}_{n}=z_{n}+\left[\sum_{j, k=1}^{n} \frac{\partial^{2} \rho}{\partial z_{j} \partial z_{k}}(0) z_{j} z_{k}-2 \sum_{j=1}^{n-1} \frac{\partial^{2} \rho}{\partial z_{j} \partial \bar{z}_{n}}(0) z_{j} z_{n}\right], \\
& \hat{z}_{j}=z_{j}, \quad 1 \leq j \leq n-1 .
\end{aligned}
$$


Relabeling $\hat{z}$ as $z$, we obtain

$$
\rho(z)=\operatorname{Re} z_{n}+\sum_{j, k=1}^{n-1} \frac{\partial^{2} \rho}{\partial z_{j} \partial \bar{z}_{k}}(0) z_{j} \bar{z}_{k}+\left|z_{n}\right|^{2}+\mathcal{O}\left(|z|^{3}\right) .
$$

Finally, by a $\mathbf{C}$-linear change of the variables $\left(z_{1}, \ldots, z_{n-1}\right)$, we may diagonalize the second term on the right to obtain

$$
\rho(z)=\operatorname{Re} z_{n}+\sum_{j=1}^{n-1} \lambda_{j}\left|z_{j}\right|^{2}+\left|z_{n}\right|^{2}+\mathcal{O}\left(|z|^{3}\right),
$$

where $\lambda_{j}=+1,-1$, or 0 .

We also let $\tilde{\rho}=\rho-4 \rho^{2}$. Note that $\tilde{\rho}$ is also a defining function for $M$ and

$$
\tilde{\rho}(z)=\operatorname{Re} z_{n}+\sum_{j=1}^{n-1} \lambda_{j}\left|z_{j}\right|^{2}-\left|z_{n}\right|^{2}-2 \operatorname{Re}\left(z_{n}^{2}\right)+\mathcal{O}\left(|z|^{3}\right) .
$$

If we let $D^{-}=\{z ; \rho(z)<0\}$ and $D^{+}=\{z ; \rho(z)>0\}$, then clearly both $\rho$ and $\tilde{\rho}$ are defining functions for the pair $\left(M, D^{-}\right)$and $-\rho$ and $-\tilde{\rho}$ are defining functions for the pair $\left(M, D^{+}\right)$(this is true at least in some neighborhood of the origin).

With the above choice of coordinates, $\left(z_{1}, \ldots, z_{n-1}\right)$ are the coordinates of the holomorphic tangent space of $M$ at 0 , and $\lambda_{1}, \ldots, \lambda_{n-1}$ are the eigenvalues of the Levi form of $M$ at 0 .

We now assume $M$ satisfies condition $Y(q)$ at 0 which means that there are $\min (n-q, q+1)$ eigenvalues of opposite sign or $\max (n-q, q+1)$ eigenvalues of the same sign. In the latter half of the $Y(q)$ condition we may assume these eigenvalues are positive. There are three cases associated with $Y(q)$.

Case I. There are $n-q$ positive and $n-q$ negative eigenvalues and $n-q \leq q+1$. In this case with $\rho_{1}=\rho$ we may label variables so that (3.2a) becomes

$$
\begin{aligned}
\rho_{1}(z)= & \operatorname{Re} z_{n}+\sum_{j=1}^{n-q}\left|z_{j}\right|^{2}-\sum_{j=n-q+1}^{2 n-2 q}\left|z_{j}\right|^{2} \\
& +\sum_{j=2 n-2 q+1}^{n-1} \lambda_{j}\left|z_{j}\right|^{2}+\left|z_{n}\right|^{2}+\mathcal{O}\left(|z|^{3}\right)
\end{aligned}
$$

and with $\tilde{\rho}_{1}=\tilde{\rho},(3.2 \mathrm{~b})$ becomes

$$
\begin{aligned}
\tilde{\rho}_{1}(z)= & \operatorname{Re} z_{n}+\sum_{j=1}^{n-q}\left|z_{j}\right|^{2}-\sum_{j=n-q+1}^{2 n-2 q}\left|z_{j}\right|^{2} \\
& +\sum_{j=2 n-2 q+1}^{n-1} \lambda_{j}\left|z_{j}\right|^{2}-\left|z_{n}\right|^{2}-2 \operatorname{Re}\left(z_{n}^{2}\right)+\mathcal{O}\left(|z|^{3}\right),
\end{aligned}
$$

where $\lambda_{j}= \pm 1$ or 0 . 
Case II. There are $q+1$ positive and $q+1$ negative eigenvalues and $q+1<$ $n-q$. With $\rho_{2}=\rho,(3.2 \mathrm{a})$ becomes

$$
\begin{aligned}
\rho_{2}(z)= & \operatorname{Re} z_{n}+\sum_{j=1}^{q+1}\left|z_{j}\right|^{2}-\sum_{j=q+2}^{2 q+2}\left|z_{j}\right|^{2} \\
& +\sum_{j=2 q+3}^{n-1} \lambda_{j}\left|z_{j}\right|^{2}+\left|z_{n}\right|^{2}+\mathcal{O}\left(|z|^{3}\right),
\end{aligned}
$$

and with $\tilde{\rho}_{2}=\tilde{\rho},(3.2 b)$ becomes

$$
\begin{aligned}
\tilde{\rho}_{2}(z)= & \operatorname{Re} z_{n}+\sum_{j=1}^{q+1}\left|z_{j}\right|^{2}-\sum_{j=q+2}^{2 q+2}\left|z_{j}\right|^{2} \\
& +\sum_{j=2 q+3}^{n-1} \lambda_{j}\left|z_{j}\right|^{2}-\left|z_{n}\right|^{2}-2 \operatorname{Re}\left(z_{n}^{2}\right)+\mathcal{O}\left(|z|^{3}\right),
\end{aligned}
$$

where $\lambda_{j}= \pm 1$ or 0 .

Case III. There are $t=\max (n-q, q+1)$ positive eigenvalues. With $\rho_{3}=\rho$, (3.2a) becomes

$$
\rho_{3}(z)=\operatorname{Re} z_{n}+\sum_{j=1}^{t}\left|z_{j}\right|^{2}+\sum_{j=t+1}^{n-1} \lambda_{j}\left|z_{j}\right|^{2}+\left|z_{n}\right|^{2}+\mathcal{O}\left(|z|^{3}\right),
$$

where $\lambda_{j}= \pm 1$ or 0 .

3.6 Lemma. (a) Suppose there are $n-q$ positive and $n-q$ negative eigenvalues and $n-q \leq q+1$ (Case I). Define

(3.6a)

$$
u_{j}^{1}(\zeta, z)= \begin{cases}\frac{\partial \rho_{1}}{\partial \zeta_{j}}(\zeta), & 1 \leq j \leq n-q, \\ \frac{\partial \rho_{1}}{\partial \zeta_{j}}(\zeta)+\left(\overline{\zeta_{j}-z_{j}}\right), & n-q+1 \leq j \leq n-1, \\ \frac{\partial \rho_{1}}{\partial \zeta_{n}}(\zeta), & j=n,\end{cases}
$$

$$
v_{j}^{1}(\zeta, z)= \begin{cases}\frac{-\partial \tilde{\rho}_{1}}{\partial \zeta_{j}}(\zeta)+\left(\overline{\zeta_{j}-z_{j}}\right), & 1 \leq j \leq n-q, \\ \frac{-\partial \tilde{\rho}_{1}(\zeta)}{\partial \zeta_{j}}, & n-q+1 \leq j \leq 2 n-2 q, \\ \frac{-\partial \tilde{\rho}_{1}(\zeta)}{\partial \zeta_{j}}+\left(\overline{\zeta_{j}-z_{j}}\right), & 2 n-2 q+1 \leq j \leq n-1, \\ \frac{-\partial \tilde{\rho}_{1}}{\partial \zeta_{n}}(\zeta)+\frac{1}{2} \frac{\partial^{2} \tilde{\rho}_{1}}{\partial \zeta_{n}^{2}}(\zeta)\left(\zeta_{n}-z_{n}\right), & j=n .\end{cases}
$$

Then $u^{1}, v^{1}$ are local support functions for $\left(M, D^{-}\right)$and $\left(M, D^{+}\right)$respectively at $z_{0}=0$. 
(b) Suppose there are $q+1$ positive and $q+1$ negative eigenvalues with $q+1<$ $n-q($ Case II). Define

(3.6b)

$$
\begin{aligned}
& u_{j}^{2}(\zeta, z)= \begin{cases}\frac{\partial \tilde{\rho}_{2}}{\partial z_{j}}(z)+\left(\overline{\zeta_{j}-z_{j}}\right), & 1 \leq j \leq q+1, \\
\frac{\partial \tilde{\rho}_{2}}{\partial z_{j}}(z), & q+2 \leq j \leq 2 q+2, \\
\frac{\partial \tilde{\rho}_{2}}{\partial z_{j}}(z)+\left(\overline{\zeta_{j}-z_{j}}\right), & 2 q+3 \leq j \leq n-1, \\
\frac{\partial \tilde{\rho}_{2}}{\partial z_{n}}(z)+\frac{1}{2} \frac{\partial^{2} \tilde{\rho}_{2}}{\partial z_{n}^{2}}(z)\left(\zeta_{n}-z_{n}\right), & j=n,\end{cases} \\
& v_{j}^{2}(\zeta, z)= \begin{cases}\frac{-\partial \rho_{2}}{\partial z_{j}}(z), & 1 \leq j \leq q+1 \\
\frac{-\partial \rho_{2}}{\partial z_{j}}(z)+\left(\overline{\zeta_{j}-z_{j}}\right), & q+2 \leq j \leq n-1, \\
\frac{-\partial \rho_{2}}{\partial z_{n}}(z), & j=n .\end{cases}
\end{aligned}
$$

Then $u^{2}$ and $v^{2}$ are local support functions for $\left(M, D^{-}\right)$and $\left(M, D^{+}\right)$respectively at $z_{0}=0$.

(c) Suppose there are $t=\max (n-q, q+1)$ positive eigenvalues (Case III). Define

$$
u_{j}^{3}(\zeta, z)= \begin{cases}\frac{\partial \rho_{3}}{\partial \zeta_{j}}(\zeta), & 1 \leq j \leq t \\ \frac{\partial \rho_{3}}{\partial \zeta_{j}}(\zeta)+\left(\overline{\zeta_{j}-z_{j}}\right), & t+1 \leq j \leq n-1, \\ \frac{\partial \rho_{3}}{\partial \zeta_{n}}(\zeta), & j=n,\end{cases}
$$

$$
v_{j}^{3}(\zeta, z)=-u_{j}^{3}(z, \zeta), \quad 1 \leq j \leq n .
$$

Then $u^{3}$ and $v^{3}$ are local support functions for $\left(M, D^{-}\right)$and $\left(M, D^{+}\right)$respectively at $z_{0}=0$.

Proof. (a) Let us Taylor expand $\rho_{1}(z)$ (3.3a) about the point $\zeta$. We obtain

$$
\begin{aligned}
\rho_{1}(z)= & \rho_{1}(\zeta)-2 \operatorname{Re}\left[\frac{\partial \rho_{1}}{\partial \zeta}(\zeta) \cdot(\zeta-z)\right]+\operatorname{Re}\left[\sum_{j, k=1}^{n} \frac{\partial^{2} \rho_{1}}{\partial \zeta_{j} \partial \zeta_{k}}(\zeta)\left(\zeta_{j}-z_{j}\right)\left(\zeta_{k}-z_{k}\right)\right] \\
& +\sum_{j, k=1}^{n} \frac{\partial^{2} \rho_{1}}{\partial \zeta_{j} \partial \bar{\zeta}_{k}}(\zeta)\left(\zeta_{j}-z_{j}\right)\left(\overline{\zeta_{k}-z_{k}}\right)+\mathcal{O}\left(|\zeta-z|^{3}\right) .
\end{aligned}
$$


From $(3.3 a)$ we see that $\left(\partial^{2} \rho_{1} / \partial \zeta_{j} \partial \zeta_{k}\right)(0)=0$ and hence

$$
\begin{aligned}
\rho_{1}(z) \geq & \rho_{1}(\zeta)-2 \operatorname{Re}\left[\frac{\partial \rho_{1}}{\partial \zeta}(\zeta) \cdot(\zeta-z)\right]+\sum_{j=1}^{n-q}\left|z_{j}-\zeta_{j}\right|^{2}-\sum_{j=n-q+1}^{2 n-2 q}\left|\zeta_{j}-z_{j}\right|^{2} \\
& +\sum_{j=2 n-2 q+1}^{n-1} \lambda_{j}\left|\zeta_{j}-z_{j}\right|^{2}+\left|\zeta_{n}-z_{n}\right|^{2}-C\left[|\zeta||\zeta-z|^{2}+|\zeta-z|^{3}\right],
\end{aligned}
$$

where $C$ is a uniform constant. By adding $\sum_{j=n-q+1}^{n-1} 2\left|\zeta_{j}-z_{j}\right|^{2}$ to both sides, then rearranging the terms and noting that $\left|\lambda_{j}\right| \leq 1,2 n-2 q+1 \leq j \leq n-1$, we obtain

$$
2 \operatorname{Re}\left[u^{1}(\zeta, z) \cdot(\zeta-z)\right] \geq \rho_{1}(\zeta)-\rho_{1}(z)+|\zeta-z|^{2}-C\left[|\zeta||\zeta-z|^{2}+|\zeta-z|^{3}\right] .
$$

We may now choose a neighborhood $U \ni 0$ of $\mathbf{C}^{n}$ so that

$$
2 \operatorname{Re}\left[u^{1}(\zeta, z) \cdot(\zeta-z)\right] \geq \rho_{1}(\zeta)-\rho_{1}(z)+\frac{1}{2}|\zeta-z|^{2} \quad \text { for } \zeta, z \in U
$$

which establishes $(2.4 \mathrm{a})$.

For $(2.4 b)$, we note that

$$
\begin{aligned}
\left.d_{\zeta}\left\{u^{1}(\zeta, z) \cdot(\zeta-z)\right\}\right|_{\zeta=z} & =u^{1}(z, z) \cdot d z=\frac{\partial \rho_{1}}{\partial z}(z) \cdot d z \\
& =\partial \rho_{1}(z)=\frac{1}{2}\left[d \rho_{1}(z)-i d^{c} \rho_{1}(z)\right]
\end{aligned}
$$

Therefore

$$
\left.d_{\zeta}\left\{\operatorname{Im} u^{1}(\zeta, z) \cdot(\zeta-z)\right\}\right|_{\zeta=z} \wedge d \rho_{1}(z)=-\frac{1}{2} d^{c} \rho_{1}(z) \wedge d \rho_{1}(z) \neq 0
$$

which establishes $(2.4 \mathrm{~b})$. We also note the same calculation verifies that $u^{1}$ satisfies (2.6).

For $v^{1}$, the proof is similar. We Taylor expand $\tilde{\rho}(z)$ about $\zeta$ to obtain

$$
\begin{aligned}
\tilde{\rho}_{1}(z) \leq & \tilde{\rho}_{1}(\zeta)+2 \operatorname{Re}\left[\frac{-\partial \tilde{\rho}_{1}}{\partial \zeta}(\zeta) \cdot(\zeta-z)+\frac{1}{2} \frac{\partial^{2} \tilde{\rho}_{1}}{\partial \zeta_{n}^{2}}(\zeta)\left(\zeta_{n}-z_{n}\right)^{2}\right] \\
& +\sum_{j=1}^{n-q}\left|\zeta_{j}-z_{j}\right|^{2}-\sum_{j=n-q+1}^{2 n-2 q}\left|\zeta_{j}-z_{j}\right|^{2}+\sum_{j=2 n-2 q+1}^{n-1} \lambda_{j}\left|\zeta_{j}-z_{j}\right|^{2} \\
& -\left|\zeta_{n}-z_{n}\right|^{2}+C\left[|\zeta||\zeta-z|^{2}+|\zeta-z|^{3}\right] .
\end{aligned}
$$

Note that $\left(\partial^{2} \tilde{\rho}_{1} / \partial \zeta_{n}^{2}\right)(0) \neq 0$ and so we cannot absorb this term into the error term (last term on the right). Adding

$$
2 \sum_{j=1}^{n-q}\left|\zeta_{j}-z_{j}\right|^{2}+2 \sum_{j=2 n-2 q+1}^{n-1}\left|\zeta_{j}-z_{j}\right|^{2}
$$

to both sides and using the definition of $v^{1}$ yields

$$
2 \operatorname{Re}\left[v^{1}(\zeta, z) \cdot(\zeta-z)\right] \geq\left[-\tilde{\rho}_{1}(\zeta)\right]-\left[-\tilde{\rho}_{1}(z)\right]+\frac{1}{2}|\zeta-z|^{2}
$$


for $\zeta, z \in U$, provided $U \ni 0$ is chosen sufficiently small. Noting that $-\tilde{\rho}_{1}$ is a defining function for the pair $\left(M \cap U, D^{+} \cap U\right)$, we see that (2.4a) is satisfied with $\rho=-\tilde{\rho}_{1}$. The proof of $(2.4 \mathrm{~b})$ for $v^{1}$ is the same as the proof of $(2.4 \mathrm{~b})$ for $u^{1}$.

The proof of (b) is analogous to (a). The only change is that one Taylor expands $\rho_{2}(\zeta)$ and $\tilde{\rho}_{2}(\zeta)$ about the point $z$ (instead of Taylor expanding $\rho_{1}(z)$ and $\tilde{\rho}_{1}(z)$ about the point $\zeta$ ).

The proof for (c) is analogous to (a). Note that after one has shown that $u^{3}$ is a local support function $\left(M, D^{-}\right)$at $z_{0}=0$, one can reverse the roles of $\zeta$ and $z$ and replace $\rho$ by $\left(-\rho_{3}\right)$ in (2.4a) to immediately obtain that $v^{3}$ is a local support function for $\left(M, D^{+}\right)$at $z_{0}=0$.

As stated at the end of $\S 2$, our goal is to construct support functions $u, v$ so that the corresponding terms $L_{M}^{-}(u)(f)$ and $L_{M}^{+}(v)(f)$ vanish when $f \in \mathscr{D}^{p, q}(M \cap U)$ (assuming that $M$ satisfies condition $Y(q)$ ). This is the goal of the next lemma.

3.7. Lemma. Assume $M$ satisfies condition $Y(q)$ and that $u^{1}, u^{2}, u^{3}, v^{1}, v^{2}, v^{3}$ are defined as in (3.6).

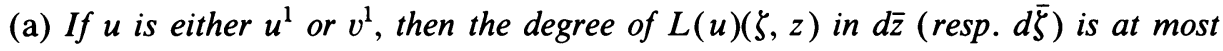
$q-1$ (resp. at least $n-q$ ).

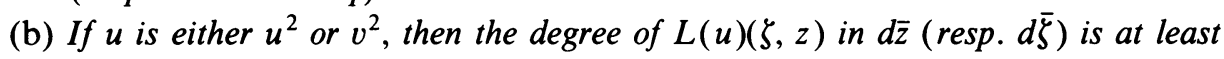
$q+1$ (resp. at most $n-q-2)$.

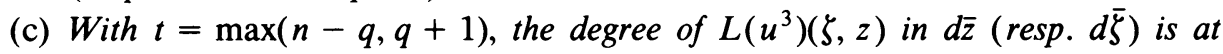
most $n-t-1$ (resp. at least $t)$. The degree of $L\left(v^{3}\right)(\zeta, z)$ in $d \bar{\zeta}($ resp. $d \bar{z})$ is at most $n-t-1$ (resp. at least $t)$.

Note that if $f \in \mathscr{D}_{M}^{p}{ }^{q}(M \cap U)$, then $f$ pairs with the piece of $L(\zeta, z)$ of type $n-q-1$ in $d \bar{\zeta}$ when integrated on $M$. Therefore in view of the above lemma we have that if $f \in \mathscr{D}_{M}^{p, q}$, then

$$
\begin{array}{ll}
L_{M}^{-}\left(u^{1}\right)(f)=L_{M}^{+}\left(v^{1}\right)(f)=0 & (\text { for Case I); } \\
L_{M}^{-}\left(u^{2}\right)(f)=L_{M}^{+}\left(v^{2}\right)(f)=0 & (\text { for Case II); } \\
L_{M}^{-}\left(u^{3}\right)(f)=L_{M}^{+}\left(v^{3}\right)(f)=0 & (\text { for Case III). }
\end{array}
$$

Proof. (a) From (3.6a) we have

$$
\begin{aligned}
& {\left[\bar{\partial} u^{1} \cdot d(\zeta-z)\right]^{n-1}=} {\left[\sum_{j=1}^{n} \bar{\partial}_{\zeta}\left(\frac{\partial \rho}{\partial \zeta_{j}}(\zeta)\right) \wedge d\left(\zeta_{j}-z_{j}\right)\right.} \\
&\left.+\sum_{j=n-q+1}^{n-1} d\left(\overline{\zeta_{j}-z_{j}}\right) \wedge d\left(\zeta_{j}-z_{j}\right)\right]^{n-1} \\
&= \sum_{k=0}^{q-1}\left(\begin{array}{c}
n-1 \\
k
\end{array}\right)\left[\sum_{j=1}^{n} \bar{\partial}_{\zeta}\left(\frac{\partial \rho}{\partial \zeta_{j}}(\zeta)\right) \wedge d\left(\zeta_{j}-z_{j}\right)\right]^{n-1-k} \\
& \wedge\left[\sum_{j=n-q+1}^{n-1} d\left(\overline{\zeta_{j}-z_{j}}\right) \wedge d\left(\zeta_{j}-z_{j}\right)\right]^{k}
\end{aligned}
$$


The first sum on the right stops at $k=q-1$ because there are only $q-1$ terms in the sum

$$
S=\sum_{j=n-q+1}^{n-1} d\left(\overline{\zeta_{j}-z_{j}}\right) \wedge d\left(\zeta_{j}-z_{j}\right)
$$

and therefore the wedge product $S^{k}$ vanishes when $k>q-1$. From (3.8) and (2.1a) it is now clear that the degree of $L\left(u^{1}\right)(\zeta, z)$ in $d \bar{z}$ is at most $q-1$. Since the total degree of $L\left(u^{1}\right)(\zeta, z)$ in $d \bar{\zeta}$ and $d \bar{z}$ is $n-1$, clearly the degree of $L\left(u^{1}\right)(\zeta, z)$ in $d \bar{\zeta}$ is at least $n-\mathrm{q}$. The proof of the other parts of the lemma are analogous.

4. The local solution to $\bar{\partial}_{M}$. It is the goal of this section to prove Theorems 1.1 and 1.3. We assume the hypersurface $M$ is presented as in $\S 3$ and that the given point $z_{0}$ in Theorem 1.1 is the origin. Let $\omega \ni 0$ be the given open set in Theorem 1.1. Choose $\omega^{\prime} \subset \subset \omega \cap U, 0 \in \omega^{\prime}$, where $U$ is the open set where the local support functions $u^{i}$, $v^{i}, i=1,2,3$, in Lemma 3.6 are defined. Choose $\phi \in C_{0}^{\infty}(\omega \cap U)$ with $\phi=1$ on $\omega^{\prime}$. Let $\tilde{f}=\phi f$, where $f \in \mathscr{E}^{p, q}(\omega)$ is the given form in Theorem 1.1.

We first assume Case I. From (2.5d) and Lemma 3.7, we have

$$
\tilde{f}=-\bar{\partial}_{M}\left\{R_{M}\left(u^{1}, v^{1}\right)(\tilde{f})\right\}-R_{M}\left(u^{1}, v^{1}\right)\left(\bar{\partial}_{M} \tilde{f}\right) \quad \text { on } M \cap U .
$$

We claim that the term

$$
\begin{aligned}
g(z) & =R\left(u^{1}, v^{1}\right)\left([M]^{0,1} \wedge \bar{\partial} \tilde{f}\right)(z) \\
& =\int_{\zeta \in M} \frac{n, n-1}{R\left(u^{1}, v^{1}\right)(\zeta, z) \wedge \bar{\partial} \tilde{f}(\zeta)}
\end{aligned}
$$

is smooth for $z$ in some open set $W \ni 0$ in $\mathbf{C}^{n}$.

To see this, note that $\bar{\partial}_{M} \tilde{f}=0$ on $\omega^{\prime}$ since $\bar{\partial}_{M} f=0$ on $\omega$. Let

$$
\varepsilon=\min \left\{|\zeta|, \zeta \in \omega-\omega^{\prime}\right\}
$$

Let

$$
W=\left\{z ; \rho_{1}(z)<\varepsilon^{2} / 8, \tilde{\rho}_{1}(z)>-\varepsilon^{2} / 8,|z|<\varepsilon / 2\right\} .
$$

If $\zeta \in \omega-\omega^{\prime}$ and $z \in W$, then from (2.4a) with $\rho=\rho_{1}$ we have

$$
\operatorname{Re}\left[u^{1}(\zeta, z) \cdot(\zeta-z)\right] \geq C\left[-\rho_{1}(z)+|\zeta-z|^{2}\right] \geq C\left[\varepsilon^{2} / 8\right]>0
$$

Likewise, from (2.4a) with $\rho=-\tilde{\rho}_{1}$ we have

$$
\begin{aligned}
\operatorname{Re}\left[v^{1}(\zeta, z) \cdot(\zeta-z)\right] & \geq C\left[\tilde{\rho}_{1}(z)+|\zeta-z|^{2}\right] \\
& \geq C \varepsilon^{2} / 8>0 \text { for } \zeta \in \omega-\omega^{\prime} \text { and } z \in W
\end{aligned}
$$

Thus $R\left(u^{1}, v^{1}\right)(\zeta, z)$ is smooth for $\zeta \in \omega-\omega^{\prime}$ and $z \in W$ and so $g$ is smooth on $W$ as desired. 
We also claim that $\bar{\partial} g=0$ on $W$. We have

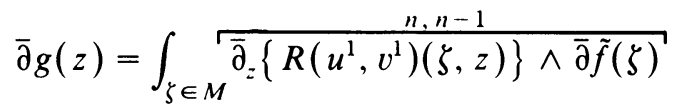

$$
\begin{aligned}
& =\int_{\zeta \in M} \sqrt{\bar{\partial}_{\zeta, z}\left\{R\left(u^{1}, v^{1}\right)(\zeta, z)\right\} \wedge \bar{\partial} \tilde{f}(\zeta)}
\end{aligned}
$$

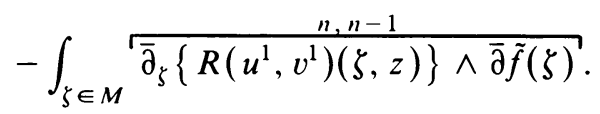

An easy integration by parts argument together with type considerations shows that the last term on the right vanishes. From (2.2) we have

$$
\bar{\partial} g(z)=\int_{\zeta \in M} \frac{n, n-1}{L\left(v^{1}\right)(\zeta, z) \wedge \bar{\partial} \tilde{f}(\zeta)}-\int_{\zeta \in M} \frac{n, n-1}{L\left(u^{1}\right)(\zeta, z) \wedge \bar{\partial} \tilde{f}(\zeta)} .
$$

Now $\bar{\partial} \tilde{f}$ is of type $(p, q+1)$ and will pair with the piece of type $(n-p, n-q-2)$ in $\zeta$, which vanishes according to Lemma 3.7(a). Likewise, the first term on the right of (4.2) vanishes. Therefore $\bar{\partial} g=0$ in $W$ as desired.

If we let $W^{\prime}$ be a ball centered at 0 with $W^{\prime} \subset \subset W$, then we can solve $\bar{\partial} u=g$ on $W^{\prime}$ with an integral kernel formula for $u$. This is well known, for example cf. Proposition 6.2 and Theorem 6.10 in [HP1]. Since $\left\{\left.g\right|_{M}\right\}_{T_{M}}$ is the second term on the right of (4.1), we have

$$
f=\tilde{f}=\bar{\partial}_{M}\left[-R_{M}\left(u^{1}, v^{1}\right)(\tilde{f})-\left(\left.u\right|_{M}\right)_{T_{M}}\right] \quad \text { on } W^{\prime} \cap M,
$$

as desired. This completes the proof of Theorem 1.1 for Case I.

For Cases II and III one may replace $u^{1}$ and $v^{1}$ by $u^{j}$ and $v^{j}$ respectively (for case $j, j=2,3)$, to obtain the analogue of (4.2), namely

$$
(4.2)^{\prime} \quad \bar{\partial} g(z)=\int_{\zeta \in M} \sqrt{L\left(v^{j}\right)(\zeta, z) \wedge \bar{\partial} \tilde{f}(\zeta)}-\int_{\zeta \in M} \sqrt{L\left(u^{j}\right)(\zeta, z) \wedge \bar{\partial} \tilde{f}(\zeta)}
$$

for case $j, j=2,3$. In these cases we still claim that $\bar{\partial} g=0$ on $W$, but this does not follow from Lemma 3.7. For example it is not a priori clear that $L\left(v^{2}\right)\left([M]^{0,1} \wedge \bar{\partial} \tilde{f}\right)$ vanishes because $\bar{\partial} \tilde{f}$ pairs with the piece of $L\left(v^{2}\right)(\zeta, z)$ of type $(n-p, n-q-2)$ in $\zeta$ which does not necessarily vanish from Lemma 3.7 (b). From (3.6bb) and (2.1), the piece of $L\left(v^{2}\right)(\zeta, z)$ of type $n-q-2$ in $d \bar{\zeta}$ equals $K\left(\zeta^{\prime}, \zeta^{\prime \prime}, \zeta_{n}, z\right) \wedge d \bar{\zeta}^{\prime \prime}$, where $\zeta^{\prime}=\left(\zeta_{1}, \ldots, \zeta_{q+1}\right), \zeta^{\prime \prime}=\left(\zeta_{q+2}, \ldots, \zeta_{n-1}\right), d \bar{\zeta}^{\prime \prime}=d \bar{\zeta}_{q+2} \wedge \cdots \wedge d \bar{\zeta}_{n-1}$ and where $K\left(\zeta^{\prime}, \zeta^{\prime \prime}, \zeta_{n}, z\right)$ is a differential form with coefficients which are analytic in $\zeta^{\prime}, \zeta_{n}$ (away from the singular set of $L\left(v^{2}\right)$ ). Therefore

$$
L\left(v^{2}\right)\left([M]^{0,1} \wedge \bar{\partial} \tilde{f}\right)(z)=\int_{\zeta \in M} K\left(\zeta^{\prime}, \zeta^{\prime \prime}, \zeta_{n}, z\right) \wedge \bar{\partial} \tilde{f}(\zeta) \wedge d \bar{\zeta}^{\prime \prime} .
$$

If we could integrate by parts in the above formula, we could easily conclude that $L\left(v^{2}\right)\left([M]^{0.1} \wedge \bar{\partial} \tilde{f}\right)=0$ on $W$ because formally $\bar{\partial}\left\{K\left(\zeta^{\prime}, \zeta^{\prime \prime}, \zeta_{n}, z\right) \wedge d \bar{\zeta}^{\prime \prime}\right\}=0$. However, $K\left(\zeta^{\prime}, \zeta^{\prime \prime}, \zeta_{n}, z\right)$ is not smooth for $\zeta \in M$ and $z \in W$. Instead, we must first 
perform the approximation argument in [B2], which is a modification of an argument used by Henkin [He] in the strictly pseudoconvex case. let

To implement the argument, we must first carefully choose the sets $\omega^{\prime}$ and $W$. We

$$
r(z)=\operatorname{Re} z_{n}-2\left|z^{\prime \prime}\right|^{2}
$$

For $\alpha>0$, let $\omega_{\alpha}=\{z \in M ; r(z)>-\alpha\}$. We claim the collection $\left\{\omega_{\alpha} ; \alpha>0\right\}$ forms a neighborhood basis for $M$ about 0 . To see this note that if $z \in M$, then $\rho_{2}(z)=0$, i.e.

$$
\operatorname{Re} z_{n}=-\left|z^{\prime}\right|^{2}+\sum_{j=q+2}^{n-1} \mu_{j}\left|z_{j}\right|^{2}-\left|z_{n}\right|^{2}+\mathcal{O}\left(|z|^{3}\right)
$$

where $\mu_{j}= \pm 1$ or 0 (cf. (3.4a)). If $z \in \omega_{\alpha}$, then $\operatorname{Re} z_{n}+\alpha>2\left|z^{\prime \prime}\right|^{2}$. Subtracting these two inequalities yields

$$
\alpha>\left|z^{\prime}\right|^{2}+\left|z^{\prime \prime}\right|^{2}+\left|z_{n}\right|^{2}-C|z|^{3} \text { for } z \in \omega_{\alpha},
$$

where $C$ is a uniform constant. It is now clear that $z \rightarrow 0$ as $\alpha \rightarrow 0$ as desired.

Now first choose $\beta>0$ so that $\omega_{\beta} \subset \subset \omega \cap \mathrm{U}$. Then choose $\alpha, 0<\alpha<\beta$. Choose $\phi \in C_{0}^{\infty}\left(\omega_{\beta}\right)$ with $\phi=1$ on $\omega_{\alpha}$ and let $\tilde{f}=\phi f$. Clearly supp $\bar{\partial}_{M} \tilde{f} \subset \omega_{\beta}-\omega_{\alpha}$. Since

$$
\operatorname{Re}\left[v^{2}(\zeta, z) \cdot(\zeta-z)\right] \geq C\left[\rho_{2}(z)-\rho_{2}(\zeta)+|\zeta-z|^{2}\right]
$$

we may choose an open set $W \ni 0$ in $\mathbf{C}^{n}$ as above such that

$$
\operatorname{Re}\left[v^{2}(\zeta, z) \cdot(\zeta-z)\right]>0
$$

for $\zeta \in \mathbf{C}^{n}$ with $-\beta \leq \rho(\zeta) \leq-\alpha$ and for $z \in W$.

Let $\pi: \mathbf{C}^{n} \rightarrow \mathbf{C}^{n-q-2}$ be the standard projection map $\pi\left(\zeta^{\prime}, \zeta^{\prime \prime}, \zeta_{n}\right)=\zeta^{\prime \prime}$. Let $A=\pi\left\{M \cap \operatorname{supp} \bar{\partial}_{M} \tilde{f}\right\} \subset \mathbf{C}^{n-q-2}$. For each $\varepsilon>0$ choose $\zeta_{j}^{\varepsilon} \in A, j=1, \ldots, N(\varepsilon)$, such that $A \subset \bigcup_{j=1}^{N(\varepsilon)} B_{j}^{\varepsilon}$, where $B_{j}^{\varepsilon}$ is the open ball of radius $\varepsilon$ in $\mathrm{C}^{n-q-2}$ centered at $\zeta_{j}^{\xi}$. Let $\left.\phi_{j}^{\varepsilon}\left(\zeta^{\prime \prime}\right)\right|_{j=1} ^{N(\varepsilon)}$ be a partition of unity for the set $A$ subordinate to the cover $\left.B_{j}^{\varepsilon}\right|_{j=1} ^{N(\varepsilon)}$. Fix any $z \in W$. By (4.5) clearly $K\left(\zeta^{\prime}, \zeta^{\prime \prime}, \zeta_{n}, z\right)$ (which is made up from coefficients of the form $L\left(v^{2}\right)$ ) is continuous for $\zeta$ in a neighborhood of supp $\bar{\partial} \tilde{f} \subset$ $\omega_{\beta}-\omega_{\alpha}$. Therefore we can approximate the integral on the right side of (4.3) by the expression

$$
\sum_{j=1}^{N(\varepsilon)} \int_{\zeta \in\left\{C^{q+2} \times B_{j}^{\varepsilon}\right\} \cap M} \phi_{j}^{\varepsilon}\left(\zeta^{\prime \prime}\right) K\left(\zeta^{\prime}, \zeta_{j}^{\varepsilon}, \zeta_{n}, z\right) \wedge \bar{\partial} \tilde{f}(\zeta) \wedge d \bar{\zeta}^{\prime \prime}
$$

for suitably small $\varepsilon$. Therefore it suffices to show that

$$
\int_{\zeta \in\left\{C^{4+2} \times B_{j}^{\varepsilon}\right\} \cap M} \phi_{j}^{\varepsilon}\left(\zeta^{\prime \prime}\right) K\left(\zeta^{\prime}, \zeta_{j}^{\varepsilon}, \zeta_{n}, z\right) \wedge \bar{\partial} \tilde{f}(\zeta) \wedge d \bar{\zeta}^{\prime \prime}=0
$$

for $\varepsilon>0$ sufficiently small and $1 \leq j \leq N(\varepsilon)$.

Now supp $\bar{\partial}_{M} \tilde{f} \subset \omega_{\beta}-\omega_{\alpha}$. Moreover it is clear from the formulas for $\rho_{2}$ and $r$ (cf. (3.4a) and (4.4)) that the set

$$
\tilde{B}_{j}^{\varepsilon}:=\left\{\left(\zeta^{\prime}, \zeta_{n}\right) \in \mathbf{C}^{q+1} \times \mathbf{C} ;-\beta \leq r\left(\zeta^{\prime}, \zeta_{j}^{\varepsilon}, \zeta_{n}\right) \leq-\alpha \text { and } \rho_{2}\left(\zeta^{\prime}, \zeta_{j}^{\varepsilon}, \zeta_{n}\right) \leq 0\right\}
$$


is a convex compact set in $\mathbf{C}^{q+2}$. If $z \in W$ is fixed, then it is now clear from (2.1), (3.6bb) and (4.5) that $K\left(\zeta^{\prime}, \zeta_{j}^{\varepsilon}, \zeta_{n}, z\right)$ is analytic for $\left(\zeta^{\prime}, \zeta_{n}\right)$ in a neighborhood of the polynomially convex set $\tilde{B}_{j}^{\varepsilon}$. Therefore, for each $\varepsilon>0$ fixed and sufficiently small, there is a sequence $\left.K_{\nu}^{j}\right|_{\nu=1} ^{\infty}$ which are entire functions in $\mathbf{C}^{q+2}$ with $K_{\nu}^{j}\left(\zeta^{\prime}, \zeta_{n}\right) \rightarrow$ $K\left(\zeta^{\prime}, \zeta_{j}^{\varepsilon}, \zeta_{n}, z\right)$ uniformly for $\left(\zeta, \zeta_{n}\right)$ in some neighborhood of $\tilde{B}_{j}^{\varepsilon}$. Thus we have

$$
\begin{aligned}
\int_{\zeta \in\left\{\mathbf{C}^{q+2} \times B_{j}^{f}\right\} \cap M} \phi_{j}^{\varepsilon}\left(\zeta^{\prime \prime}\right) K\left(\zeta^{\prime}, \zeta_{j}^{\varepsilon}, \zeta_{n}, z\right) \wedge \bar{\partial} \tilde{f}(\zeta) \wedge d \bar{\zeta}^{\prime \prime} \\
\quad=\lim _{\nu \rightarrow \infty} \int_{\zeta \in\left\{\mathbf{C}^{q+2} \times B_{j}^{\varepsilon}\right) \cap M} \phi_{j}^{\varepsilon}\left(\zeta^{\prime \prime}\right) K_{\nu}^{j}\left(\zeta^{\prime}, \zeta_{n}\right) \wedge \bar{\partial} \tilde{f}(\zeta) \wedge d \bar{\zeta}^{\prime \prime} .
\end{aligned}
$$

For each $\nu>0$, we may integrate by parts in the last integral and conclude that the terms in the above limit vanish because

$$
\bar{\partial}\left\{K_{\nu}^{j}\left(\zeta^{\prime}, \zeta_{n}\right)\right\}=0 \quad \text { and } \bar{\partial}\left\{\phi_{j}^{\varepsilon}\left(\zeta^{\prime \prime}\right) d \bar{\zeta}^{\prime \prime}\right\}=0 .
$$

Thus $L\left(v^{2}\right)\left([M]^{0,1} \wedge \bar{\partial} \tilde{f}\right)(z)=0$ for $z \in W$ as desired. Similar arguments show that $L\left(u^{2}\right)\left([M]^{0,1} \wedge \bar{\partial} \tilde{f}\right)$ and $L\left(v^{3}\right)\left([M]^{0,1} \wedge \bar{\partial} \tilde{f}\right)$ vanish on an appropriately chosen neighborhood $W \ni 0$ in $\mathbf{C}^{n}$. Note that $L\left(u^{3}\right)\left([M]^{0,1} \wedge \bar{\partial} \tilde{f}\right)=0$ by type considerations (this is analogous to $L\left(u^{1}\right)$ and $L\left(v^{1}\right)$ as done above).

From (4.2)' we conclude that $\bar{\partial} g=0$ on $W$ in Cases II or III as well as Case I. The rest of the proof for Cases II or III is the same as the proof for Case I.

For the proof of Theorem 1.3, note that condition $Y(0)$ reduces to Case II only, i.e. there is at least one positive and one negative eigenvalue in the Levi form of $M$ at 0 . Equation (4.1) with $u^{1}$ and $v^{1}$ replaced by $u^{2}$ and $v^{2}$ yields

$$
f=\tilde{f}=-R_{M}\left(u^{2}, v^{2}\right)\left(\bar{\partial}_{M} \tilde{f}\right) \text { on } \omega^{\prime} .
$$

This holds whenever $\bar{\partial}_{M} f=0$ on $\omega^{\prime}$, i.e. $f$ is CR on $\omega^{\prime}$. The above arguments show that $-R_{M}\left(u^{2}, v^{2}\right)\left(\bar{\partial}_{M} \tilde{f}\right)$ and hence $f$ is the restriction on $M \cap W$ of the form

$$
-R\left(u^{2}, v^{2}\right)\left([M]^{0,1} \wedge \bar{\partial} \tilde{f}\right)
$$

which was shown to be $\bar{\partial}$ closed, i.e. holomorphic, in $W$. This establishes Theorem 1.3 with (4.6) giving an explicit formula for the CR extension.

5. Nonsolvabilty of $\bar{\partial}_{b}$. In this section, we prove Theorem 1.2 by kernel methods. If $M$ is strictly pseudoconvex, then nonsolvability at the top degree $(q=n-1)$ has been thoroughly analyzed by Henkin (cf. [He, Theorem 4.1]). We shall use his results to prove Theorem 1.2.

Let us assume $q<n-1$ and $z_{0}=0$. In this case, we let $\rho$ be a defining function for $\left(M, D^{-}\right)$, where

$$
\rho(z)=\operatorname{Re} z_{n}+\left|z^{\prime}\right|^{2}-\left|z^{\prime \prime}\right|^{2}+\mathcal{O}\left(|z|^{3}\right)
$$

where we have written $z^{\prime}=\left(z_{1}, \ldots, z_{q}, z_{n}\right)$ and $z^{\prime \prime}=\left(z_{q+1}, \ldots, z_{n-1}\right)$. Set

$$
\begin{aligned}
& D_{0}^{-}:=\left\{z^{\prime} \in \mathbf{C}^{q+1} ; \rho\left(z^{\prime}, 0^{\prime \prime}\right)<0\right\}, \\
& D_{0}^{+}:=\left\{z^{\prime} \in \mathbf{C}^{q+1} ; \rho\left(z^{\prime}, 0^{\prime \prime}\right)>0\right\} .
\end{aligned}
$$


The set $D_{0}$ is clearly strictly convex (near 0 ) with boundary $M_{0}=\left\{z^{\prime} \in\right.$ $\left.\mathrm{C}^{q+1} ; \rho\left(z^{\prime}, 0\right)=0\right\}$. Since the Levi form of $M_{0} \subset C^{q+1}$ at 0 has $q$ positive eigenvalues, we may use Lemma 3.6(c) with $n$ replaced by $q+1$ and $t$ replaced by $q$ to obtain local support functions $u$ and $v$ for $\left(M_{0}, D_{0}^{-}\right)$and $\left(M_{0}, D_{0}^{+}\right)$, respectively, where

$$
u_{j}\left(\zeta^{\prime}, z^{\prime}\right)=\frac{\partial \rho}{\partial \zeta_{j}}\left(\zeta^{\prime}, 0^{\prime \prime}\right), \quad v_{j}\left(\zeta^{\prime}, z^{\prime}\right)=-\frac{\partial \rho}{\partial z_{j}}\left(z^{\prime}, 0^{\prime \prime}\right), \quad 1 \leq j \leq q+1 .
$$

Let $\alpha \in \mathscr{D}_{M_{0}}^{0 . q}\left(U \cap M_{0}\right)$, where $U \ni 0$ is the open set in $\mathbf{C}^{n}$ where $u$ and $v$ are defined. From equation ( $2.5 \mathrm{~d})$ we have

$$
\alpha=-\bar{\partial}_{M_{0}}\left\{R_{M_{0}}(u, v)(\alpha)\right\}+L_{M_{0}}^{+}(v)(\alpha) \quad \text { on } M_{0} \cap U .
$$

Since $M_{0} \cap U$ is strictly convex we can choose $\alpha \in \mathscr{D}_{M_{0}^{0}}^{0, q}\left(M_{0} \cap U\right)$ (cf. [He, Theorem 4.2]) so that the equation $\bar{\partial}_{M_{0}} g=\alpha$ has no continuous solution on any neighborhood of $0^{\prime}$ in $M_{0}$.

Let

$$
\begin{aligned}
f_{0}\left(z^{\prime}\right) & =L(v)\left(\left[M_{0}\right]^{0,1} \wedge \alpha\right)\left(z^{\prime}\right) \\
& =\int_{\zeta^{\prime} \in M_{0}} L(v)\left(\zeta^{\prime}, z^{\prime}\right) \wedge \alpha\left(\zeta^{\prime}\right) .
\end{aligned}
$$

By Proposition 2.5(a), $f_{0}$ is smooth on $\overline{D_{0}^{+}} \cap U$. We claim that $\bar{\partial}_{z^{\prime}} f=0$ on $\overline{D_{0}^{+}} \cap U$. Note that if $z^{\prime} \in D_{0}^{+} \cap U$, then

$$
\begin{aligned}
\bar{\partial}_{z^{\prime}} f\left(z^{\prime}\right) & =\int_{\zeta^{\prime} \in M_{0}} \sqrt{\bar{\partial}_{z^{\prime}}\left\{L(v)\left(\zeta^{\prime}, z^{\prime}\right)\right\} \wedge \alpha\left(\zeta^{\prime}\right)} \\
& =\int_{\zeta^{\prime} \in M_{0}} \sqrt{\bar{\partial}_{\zeta^{\prime}, z^{\prime}} L(v)\left(\zeta^{\prime}, z^{\prime}\right) \wedge \alpha\left(\zeta^{\prime}\right)} .
\end{aligned}
$$

The last equality holds because $v$ is independent of $\zeta^{\prime}$ and therefore $L(v)\left(\zeta^{\prime}, z^{\prime}\right)$ is analytic in $\zeta^{\prime}$. The above expression vanishes by (2.2). Thus $\bar{\partial}_{z^{\prime}} f_{0}=0$ on $\bar{D}_{0}^{+} \cap U$ as desired.

Now let $\pi\left(z^{\prime}, z^{\prime \prime}\right)=z^{\prime}$ and set $\tilde{f}=\pi^{*} f_{0}$, i.e. $\tilde{f}$ is just the extension of $f_{0}$ with $\tilde{f}$ independent of $z^{\prime \prime}$. Clearly, $\tilde{f}$ is smooth and $\bar{\partial} \tilde{f}=0$ on $\left\{\overline{D_{0}^{+}} \cap U\right\} \times \mathbf{C}^{n-q-1}$. In addition, since the Levi form of $M$ has negative eigenvalues in the directions $z^{\prime \prime} \in \mathbf{C}^{n-q-1}$, clearly $M \cap U \subset\left\{\overline{D_{0}^{+}} \cap U\right\} \times \mathbf{C}^{n-q-1}$ if $U \ni 0$ is chosen sufficiently small. We conclude that $\bar{\partial}_{M} f=0$ on $M \cap U$, where $f=\left[\left.\tilde{f}\right|_{M}\right]_{T_{M}}$. We claim that $\bar{\partial}_{M} g=f$ has no continuous solution $g$ in any neighborhood of the origin in $M$. Note that if $\bar{\partial}_{M} g=f$ on $M$ near 0 , then by restricting to $M_{0}$, we have

$$
\bar{\partial}_{M_{0}} g_{0}=L_{M_{0}}^{+}(v)(\alpha) \text { on } M_{0} \text { near } 0 \text {. }
$$

However, this equation together with (5.1) implies that the equation $\bar{\partial}_{M_{0}} u=\alpha$ is solvable on some neighborhood of 0 in $M_{0}$, and this contradicts the choice of $\alpha$.

This completes the nonsolvablility in degree $q$. To obtain nonsolvability in degree $n-q-1$, one repeats the above arguments except one lets $M_{0}$ be the slice of $M$ with $z_{1}=\cdots=z_{q}=0$. 
We note that nondegeneracy of the Levi form is not necessary in the above arguments for nonsolvability in degree $q$. All that is needed is for the Levi form to have $q$ positive eigenvalues (so that $M$ is strictly convex) and we need $M \cap U \subset$ $\left.\left\{\overline{D_{0}^{+}} \cap U\right\} \times \mathbf{C}^{n-q-1}\right\}$. For example, the above arguments can apply to $M=$ $\{z ; \rho(z)=0\}$, where $\rho(z)=\operatorname{Re} z_{n}+\left|z^{\prime}\right|^{2}-\phi\left(z^{\prime \prime}\right)$ with $\phi\left(z^{\prime \prime}\right)$ smooth and $\phi\left(z^{\prime \prime}\right) \geq$ 0 . We also remark that Treves [T2] has recently obtained nice nonsolvability results assuming $M$ is real analytic.

\section{REFERENCES}

[AH] A. Andreotti and C. D. Hill, E. E. Levi convexity and the Hans Lewy problem. I, II, Ann. Scuola Norm. Sup. Pisa Cl. Sci. 26 (1972), 325-363; ibid. 26 (1972), 747-806.

[B1] A. Boggess, Kernels for the tangential Cauchy Riemann equations, Trans. Amer. Math. Soc. 269 (1980), 1-49.

[B2] __ Kernels for the local solvability of the tangential Cauchy Riemann equations, Duke Math. J. 47 (1980), 903-921.

[HP1] R. Harvey and J. Polking, Fundamental solutions in complex analysis. I, Duke Math. J. 46 (1979), $253-300$

[HP2] _ Fundamental solutions in complex analysis. II, Duke Math. J. 46 (1979), 301-340.

[Hi] C. D. Hill, A hierarchy of nonsolvability examples, Proc. Sympos. Pure Math., vol. 27, Amer. Math. Soc., Providence, R. I., 1975, pp. 301-305.

[He] G. M. Henkin, The Lewy equation and analysis on pseudo convex manifolds, Uspheki Mat. Nauk 32 (1977): English transl., Russian Math. Surveys 32 (1977), 59-130.

[FK] G. B. Folland and J. J. Kohn, The Neumann problem for the Cauchy Riemann complex, Princeton Univ. Press, Princeton, N. J., 1972.

[L] H. Lewy, On the local character of the solution of an atypical differential equation in three variables and a related problem for regular functions of two complex variables, Ann. of Math. (2) 64 (1956), 514-522.

[T1] F. Treves, $A$ remark on the Poincare lemma in analytic complexes with nondegenerate Levi form (preprint).

[T2] _ On the local solvabilty and the local integrability of systems of vector fields, Acta Math. 151 (1983), 1-48.

Department of Mathematics. Texas A \& M University, College Station, Texas 77840 